\title{
Ab-initio dynamics of Gas-phase and Aqueous-phase Hydrolysis of Adenosine Triphosphate
}

\author{
Raghav Saxena ${ }^{1}$, Phani Kumar Avanigadda ${ }^{1}$, Raghvendra Singh $^{1}$, and Vishal Agarwal ${ }^{1}$ \\ ${ }^{1}$ Indian Institute of Technology Kanpur
}

November 2, 2020

\begin{abstract}
Adenosine triphosphate (ATP) hydrolysis is a well-known biological reaction which plays an important role in many biological processes. In this study, we have modelled the non-enzymatic hydrolysis of ATP in the gas-phase and the aqueous-phase by performing $a b$ initio molecular dynamics simulations combined with an enhanced sampling technique. In the gas-phase, we studied hydrolysis of fully protonated ATP molecule and in the aqueous-phase, we studied hydrolysis of ATP coordinated with: a) two $\mathrm{H}^{+}$ions (H-ATP), b) $\mathrm{Mg}^{2+}\left(\mathrm{Mg}\right.$-ATP) and c) $\mathrm{Ca}^{2+}$ (Ca-ATP). We show that gas-phase ATP hydrolysis follows a two-step dissociative mechanism via a highly stable metaphosphate intermediate. The Adenine group of the ATP molecule plays a crucial role of a general base; temporarily accepting protons and, thus helping in the elimination-addition process. In the aqueous-phase hydrolysis of ATP, we find that the cage of solvent molecules increases the stability of the terminal phosphoanhydride bond through a well-known cage-effect. Further, we find that the aqueous-phase hydrolysis happens with the help of nearby water molecules, which assumes the role of a base assisting in proton diffusion through Grotthuss mechanism. We obtained much lower free-energy barriers for the aqueous-phase hydrolysis of ATP coordinated with divalent ions $\left(\mathrm{Mg}^{2+}\right.$ and $\mathrm{Ca}^{2+}$ ) compared to hydrolysis of ATP coordinated with only $\mathrm{H}^{+}$ions, suggesting a clear catalytic effect of the divalent ions. We find a single-step dissociative-type mechanism for Mg-ATP, while we find a $\mathrm{S}_{\mathrm{N}}$-2-type concerted hydrolysis pathway for Ca-ATP.
\end{abstract}

\section{Hosted file}

Atp_hyd7.pdf available at https://authorea.com/users/372267/articles/490371-ab-initiodynamics-of-gas-phase-and-aqueous-phase-hydrolysis-of-adenosine-triphosphate 